\title{
Down the Block and Around the Corner \\ The Impact of Radio Propagation on Inter-vehicle Wireless Communication
}

\author{
John S. Otto, Fabián E. Bustamante, and Randall A. Berry \\ Department of Electrical Engineering and Computer Science \\ Northwestern University \\ Evanston, Illinois, United States \\ $\{$ jotto,fabianb,rberry\}@eecs.northwestern.edu
}

\begin{abstract}
Vehicular networks are emerging as a new distributed system environment with myriad possible applications. Most studies on vehicular networks are carried out via simulation, given the logistical and economical problems with largescale deployments. This paper investigates the impact of realistic radio propagation settings on the evaluation of VANET-based systems. Using a set of instrumented cars, we collected IEEE 802.11 b signal propagation measurements between vehicles in a variety of urban and suburban environments. We found that signal propagation between vehicles varies in different settings, especially between lineof-sight ("down the block") and non line-of-sight ("around the corner") communication in the same setting. Using a probabilistic shadowing model, we evaluate the impact of different parameter settings on the performance of an epidemic data dissemination protocol and discuss the implications of our findings. We also suggest a variation of a basic signal propagation model that incorporates additional realism without sacrificing scalability by taking advantage of environmental information, including node locations and street information.
\end{abstract}

\section{Introduction}

Vehicular networks are emerging as a new distributed system environment with myriad possible applications that range from traffic information systems and road safety [1][5] to urban sensing and entertainment [6]. Vehicular adhoc networks (VANETs) provide infrastructureless, rapidly deployable, self-configurable network connectivity. The network is made of vehicles interconnected by wireless links and willing to store and forward data for their peers. As vehicles move freely and organize themselves arbitrarily, message routing is done dynamically based on network connectivity. Compared with other ad-hoc networks, VANETs are particularly challenging due in part to the vehicles' high rate of mobility and the numerous signal-weakening obstructions, such as buildings, in their environments.
Because of logistic and economic limitations, most studies on mobile ad-hoc networks are carried out via simulation, typically using models of wireless networking components layered above a discrete event simulator. Previous work has pointed out that many of these models may be based on "too simplistic" assumptions [7], [8] and some have tried to quantify the potential risks of such assumptions (e.g. about radio propagation models [9]-[11] or node mobility patterns [12], [13]). While more complex and realistic models are available (e.g. McKown and Hamilton's ray-tracing [14]), their use is limited to relatively small networks and not practical for evaluating typically large-scale VANETs.

This paper investigates the impact of more realistic configurations for radio propagation models on the evaluation of VANET-based systems. Using a set of instrumented vehicles, we experimentally characterize IEEE 802.11 b signal propagation between vehicles in a variety of urban and suburban areas in the Chicago area. We found that signal propagation varies depending on the environment, and especially between line-of-sight (LOS) and non-LOS ("Around-theCorner"-ATC) communication in the same environment. For instance, we found that the effective communication window for two vehicles in an urban setting can be nearly $35 \%$ shorter than that in an open field (45 sec. to $70 \mathrm{sec}$.). Total throughput can vary between settings by as much as $42 \%$ (1.9 $\mathrm{MB}$ in an urban setting and 3.3 $\mathrm{MB}$ in an open field).

To understand the implications of these variations on the performance of common VANET applications, we evaluate a simple epidemic protocol using parameter settings derived from our measurements. Epidemic-based data dissemination protocols are fairly well understood and commonly adopted by many applications in this domain [15]. For our study, we employ the JiST/SWANS wireless simulator [16] and rely on STRAW [13] to model vehicular mobility. We model radio propagation using the JiST/SWANS implementation of the probabilistic shadowing model with log-distance path loss [17].

We show that, while a single parameter setting may be sufficient to model simple, open-field like environments, it is not enough to accurately characterize more complex settings. In an open field environment, the ATC and LOS path loss 
exponents are nearly identical (3.35 and 3.31) and the minor difference has no impact on the performance of the evaluated epidemic protocol. In more complex urban environments, on the other hand, the ATC and LOS path loss exponent vary by nearly $22 \%$ (4.05 and 3.17, respectively). This difference results in an 8x increase in median delivery latency for our basic epidemic protocol when employing ATC instead of LOS parameter settings (133 vs. 15 seconds).

We propose a compromise solution for modeling signal propagation in VANET settings without sacrificing scalability. Our approach takes advantage of node location information and urban road maps to select between two parameter settings based on the position of the communicating vehicles - in line of sight or around the corner.

After a brief overview of the context of our work in Section 2, we describes the setup of our experimental measurement effort in Section 3 and analyze the radio propagation characteristics measured in Section 4. We study the implications of our findings on simulation-based studies of application performance in Section 6, after describing our evaluation setting in Section 5. We review related work and conclude in Sections 7 and 8.

\section{Background}

The performance characteristics of the network stack's underlying physical layer define the boundaries of a system's abilities. In wired networks, the characteristics of the physical layer are well understood and easily modeled. Indeed, for modern wired networks, the physical layer is well modeled as simply a fixed-rate "bit pipe," which allows for it to be easily integrated into network simulators. In wireless networks, accurately modeling the physical layer is complicated by the intricacies of radio propagation as well as the mutual interference among the nodes in the network. As it has been pointed out, inaccurate modeling assumptions can lead to poor estimates of overall performance [7]-[11].

Most VANET simulators (e.g. ns-2 [18], JiST/SWANS [16] and GloMoSim [19]) implement the free space, two-ray ground reflection and probabilistic shadowing models. The first two are deterministic models that provide received signal power at a given distance on a flat terrain. The free space model assumes LOS with ideal propagation, while the two-ray ground model accounts for the signal reflecting off the ground. Neither of these models capture the characteristics of typical VANET settings with complex obstacle patterns. The probabilistic shadowing model, on the other hand, uses a statistical approach that takes into account the effects of obstacles on propagation.

An alternative is to resort to more complex approaches, such as ray-tracing by McKown and Hamilton [14]. Such models require a database containing the exact locations of all obstructions and terrain features. The received signal strength is then determined by accounting for the reflection (as well as diffraction and scattering) of the transmitted signal on the obstructions. This approach accurately models the effect of obstructions; however, it is prohibitively expensive in terms of computation and clearly infeasible for experimentation with large scale VANETs.

Thus, for this work we rely on the probabilistic shadowing model with log-distance path loss [17], following [12]'s argument that this probabilistic model will better capture the real-world characteristics of radios and thus yield better estimates of system performance. In this model, originally proposed by Lee [20], the mean received signal strength decays with distance according to a power-law path loss model, where the path loss exponent can be configured depending on the setting. In addition to the deterministic path loss, the shadowing model also includes a probabilistic term that models the effect of various obstructions in the environment. This term is a log-normal random variable around the mean of the signal strength computed by the path loss exponent. This term can be set to reflect the variance of the simulated environment. Although there have been numerous measurement studies on the appropriate parameters of this model for cellular systems in different settings [17], [20], there has been very little work in the context of VANETs. One such study used this model to characterize signal propagation in the $5.9 \mathrm{GHz}$ Dedicated Short Range Communications (DSRC) band in a suburban environment [21].

The shadowing model as well as the other models discussed above focus on "large-scale" fading. Radio signals are also subject to "small-scale" fading, which models signal fluctuations due to the interactions among copies of the transmitted signals that are received over multiple paths [17]. In the context of VANETs, variations in signal strength due to small-scale fading are minor when compared to those due to obstacles and are, thus, not explicitly modeled. Any small-scale fading effects in our measurements are captured in the resulting empirical shadowing parameter.

\section{Measurement Configuration}

We employed a set of equipped vehicles to study the radio propagation characteristics of typical urban settings. In the following paragraphs, we describe the equipment used, the different environments in which we collected measurements, and our experiment model.

\subsection{Equipment}

Figure 1 shows our nodes, which consist of Soekris net4801-60 machines with an Ubiquiti Networks SuperRange2 2.4 GHz 802.11b/g mini-PCI module for wireless communications attached to a Pacific Wireless $7 \mathrm{dBi}$ $2.4 \mathrm{GHz}$ omnidirectional antenna that is mounted on the roof of the vehicle. For positioning, we use a Garmin GPS 18 


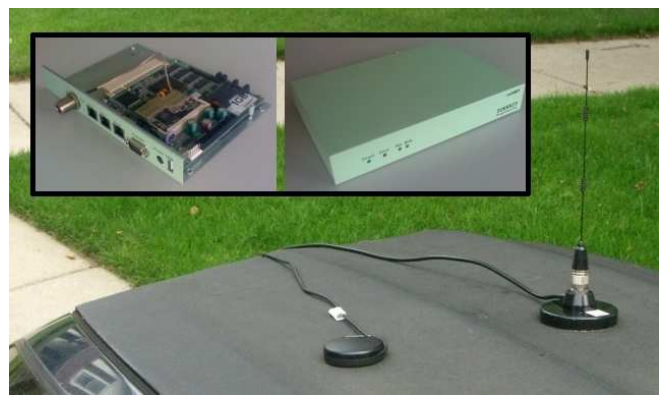

Figure 1. GPS and radio antennas installed on the roof of a vehicle. The car's windshield is visible in the lower left-hand corner of the image. The inset shows the internal hardware and exterior of the Soekris machine.

USB unit that provides new position data once per second. The GPS device is also attached to the roof.

The nodes run a 2.6.19 Linux kernel, invoke command line tools such as iperf and tcpdump, and acquire per-packet wireless statistics from the MadWifi driver.

Transmitting nodes were configured to send a $1024 \mathrm{~Kb} / \mathrm{s}$ stream of UDP packets using iperf. The wireless cards were set to operate on IEEE $802.11 \mathrm{~b}$ channel $1(2.412 \mathrm{GHz})$ in ad hoc mode with bitrate fixed at $2 \mathrm{Mb} / \mathrm{s}$, request-to-send and fragmentation disabled. We have collected data using high transmit power levels $(22-26 \mathrm{dBm})$ and found that our results are consistent across these values.

\subsection{Experimental Environments}

We collect data in three different environments: open field, suburban residential, and urban. Since beginning data collection in October 2007, we have accumulated over 36 hours of trace data across 14 sessions with myriad experimental configurations and conditions (e.g. a different hours during the day and vehicle speed).

3.2.1. Open Field. For a representative open-field setting, we selected a simple environment with almost no buildings and without obstacles around the corner where we took our measurements. Figure 2(a) shows a satellite image and road overlay for the selected location. For the line of sight measurements, two nodes drove north and south along Riverwoods Road around the intersection. For the aroundthe-corner measurements, a node was parked west of the intersection while a moving node continued to travel on Riverwoods Road.

There are trees to the north of the intersection and a park with baseball fields on the southeast corner. The largest obstacle between the nodes at any time was a small tree. The closest building, at the park, is hundreds of meters away.

At the night-time data collection, there was very light traffic-a car passed through the intersection every few min- utes. However, during the day, tens of cars were queued up in each direction at the stop sign.

3.2.2. Suburban. The second environment is a suburban residential area. This setting is more challenging than the open field because it has many obstacles, including buildings, trees and other vehicles parked on the road. There are one- and two-story houses, set back from the road, with space between the buildings.

Figure 2(b) shows the setting in which we took our suburban measurements. A node was parked north of the intersection of Maple Avenue and 5th Street on the east side of the road. LOS measurements were taken with nodes driving north and south on 5th Street. The around-the-corner experiment data was collected when the moving node was on Maple Avenue west of 5th Street. Similar to the open field environment, traffic was light at night, but heavier during the day.

3.2.3. Urban. Our final experiment setting is an urban location, defined by its large and tall buildings that are very close to the street. There are few gaps between buildings, and it is unlikely that a signal could travel over the buildings because of their height.

Figure 2(c) shows our urban environment. For the LOS experiment, nodes drove north and south on Maple Avenue, spanning 3 blocks. In the around-the-corner experiment, a node was parked east of Maple Avenue on University Place while a moving node traveled away from that intersection on Maple Avenue to the north. At night, the traffic was light, but during the early evening there was much more traffic on the road.

\subsection{Experiment Model}

Each of the experiments we conducted has three phases:

- Noise Characterization: The nodes are stationary with LOS communication. We use this data to evaluate the potential impact of noise in our measurements, which may be contributed by other radio devices or interfering appliances.

- Line-of-Sight (LOS) Communication: Nodes drive continuously up and down a 3- to 4-block region of one street. This gives predominantly line of sight communication except when other vehicles pass between the nodes. This allows analysis of the relationship between signal propagation distance and LOS signal strength.

- Around-the-Corner (ATC) Communication: One node is parked on the side of the street, while other nodes drive on an adjacent perpendicular street. The moving node has LOS while it is in the intersection, but may lose LOS as it travels away from the intersection due to obstacles. This data yields a microscopic analysis of the change in model parameters during the transition from LOS to non-LOS communication. 


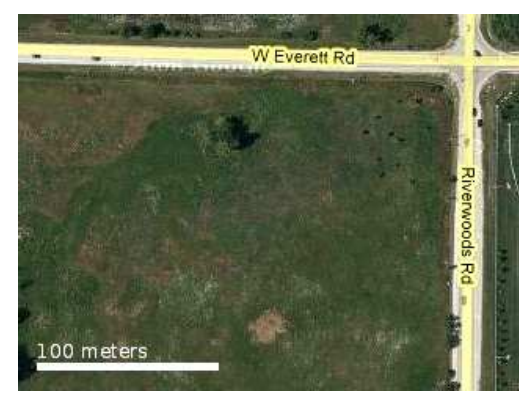

(a) Open field (Everett and Riverwoods)

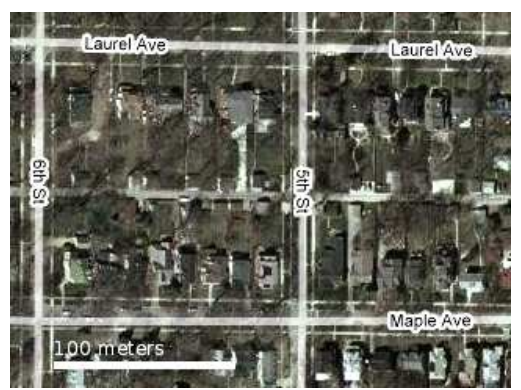

(b) Suburban (5th and Maple)

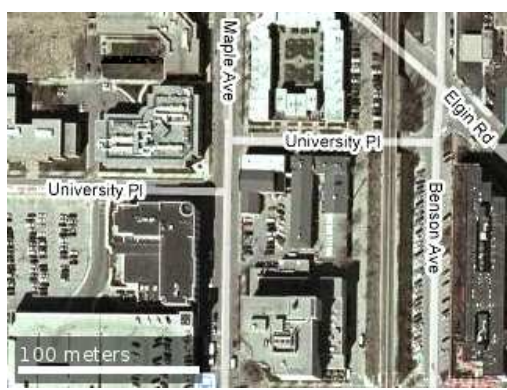

(c) Urban (University and Maple)

Figure 2. Maps of the open field, suburban and urban measurement areas. Images from Google Maps.

\section{Measurement Analysis}

In this section, we discuss our experimental findings, characterizing the impact of noise and then analyzing the effect of different environments and node position configurations.

\subsection{Noise Characterization}

A number of devices, including other IEEE 802.11 units, operate in the same bandwidth as our radios and generate radio interference. Greater use of this bandwidth, and the resulting high noise levels and variability in signal strength, is correlated with the daytime hours. Variability will also depend on the unique density and distribution of other devices and obstacles (such as other vehicles) in each environment.

To understand the impact of noise-related variations in signal strength on our subsequent analysis, we collected signal strength statistics in the measured environments at different times of the day. We monitored per-packet signal strength statistics on a constant bit-rate UDP stream while nodes were about 50 meters apart and stationary. The standard deviation of received signal strength was at most $3.26 \mathrm{~dB}$, in the urban environment in the evening with heavy traffic. Higher variations were correlated with increased traffic on the road. The lowest level of variation was recorded in the open field environment at night: $0.8 \mathrm{~dB}$. The other noise distributions have standard deviations between 1 and $2 \mathrm{~dB}$. Our radios have a receive threshold of about $-88 \mathrm{~dB}$, and we observe signal strength of about $-50 \mathrm{~dB}$ at 50 meters apart, so the possible error in signal strength due to noise variation is at most about $10 \%$, and commonly around $3-4 \%$. The minimal variations in signal strength show that noise is not a significant factor in our measurements.

\subsection{Data Interpretation}

Performing a comparative analysis between environments based on the path loss and shadowing models requires a number of calculations using wireless communication parameters as well as signal strength and distance measurements.
Post-processing of GPS data allows continuous distance estimation between the nodes. Playback of GPS traces confirms that significant GPS errors are rare in our environmental settings over the time scale of our experiments.

To highlight the differences between environments, we factor distance out of our per-packet measurements, representing each packet's signal strength measurement in terms of an effective path loss exponent (PLE). This is calculated based on a log-distance path loss model using a free space reference distance of 1 meter [17]. Wavelength $(\lambda=0.124 \mathrm{~m})$ is calculated from the frequency of the center of IEEE $802.11 \mathrm{~b}$ channel $1(f=2.412 \mathrm{GHz})$.

The radiotap header for a received packet includes absolute signal strength, or Received Signal Strength Indication $(R S S I)$ in $\mathrm{dB}$. When a packet is received, the first step is to compute the path loss value using the transmission power $\left(P_{T}=22\right.$ to $\left.26 \mathrm{dBm}\right)$, and the antenna gains of the transmitter $\left(G_{T} \mathrm{dBi}\right)$ and receiver $\left(G_{R}=7 \mathrm{dBi}\right)$.

$$
\text { path loss }(\mathrm{dB})=G_{T}+P_{T}+G_{R}-R S S I
$$

Since distance $(d)$ between the two nodes is known, the PLE $(\beta)$ can be calculated:

$$
\beta=\frac{\text { path loss }-20 \log _{10} \frac{4 \pi}{\lambda}}{10 \log _{10} d}
$$

We sort all packets into bins by distance. The median PLE observed in a particular configuration (e.g. LOS communication in the open field) is determined from the distribution of the mean PLE from each bin. The shadowing parameter $\left(\sigma_{d B}\right)$ is the standard deviation of the difference between predicted and actual path loss fading over all samples.

\subsection{Comparing Environments and Vehicle Place- ments}

In the following paragraphs, we examine the line-of-sight measurements for each of our environments. We then look at the transition from LOS to non-LOS communication.

Table 1 contains the PLE and shadowing parameters for each environment, considering either LOS communication, ATC communication, or both together ("Combined"). The 


\begin{tabular}{|c|c|c|c|c|c|c|}
\hline & \multicolumn{2}{|c|}{ LOS } & \multicolumn{2}{c|}{ ATC } & \multicolumn{2}{c|}{ Combined } \\
\hline & $\beta$ & $\sigma_{d B}$ & $\beta$ & $\sigma_{d B}$ & $\beta$ & $\sigma_{d B}$ \\
\hline Open Field & 3.31 & 4.84 & 3.35 & 4.44 & 3.50 & 5.90 \\
\hline Suburban & 3.14 & 7.28 & 3.87 & 8.44 & 3.62 & 9.40 \\
\hline Urban & 3.17 & 9.15 & 4.05 & 10.74 & 3.43 & 11.95 \\
\hline
\end{tabular}

Table 1. Median path loss exponent (PLE) and shadowing standard deviation for each configuration and environment. The open field parameters are taken from the measurements with heavy traffic on the roads. The open field combined parameter analysis includes additional measurements that increased the median observed PLE.

Combined values incorporate all measurements taken in each setting into a single set of parameters. The shadowing parameter for the combined data set is always larger than that of the LOS or ATC data sets because greater probabilistic variation is necessary to match the relatively large diversity of communication scenarios (e.g. LOS, ATC) in the environment.

4.3.1. Line-of-Sight. We first examine the changes in path loss exponent for the LOS cases in each of our environments. We expect that in an open field the path loss exponent will be consistent over a wide range of distances, with little variation at any given distance. As we move into suburban and urban settings, the increasing presence of obstacles should result in a higher shadowing parameter.

In all of our measurements, there is a significant rise in the effective PLE at distances shorter than 50 meters under varying transmit power levels, times of day, traffic conditions, and vehicle types (e.g. convertibles, sedans, pickup trucks). We attribute this effect to the existence of multiple signal propagation paths; in addition to the direct path, reflections off the roofs of the vehicles would make a significant contribution to the received signal strength. As distance increases, the proportional difference in length of the multiple paths asymptotically approaches zero, which explains why we do not see this effect at higher distances. We focus our discussion on the consistent measurements collected when nodes are at least 50 meters apart. Since this effect is only observed at very close range, even with higher than expected signal attenuation, should not prevent the packet from being received.

Open Field. Figure 3(a) shows the distribution of PLE over distance for our open field environment, both with traffic during the day and without traffic at night. The scenario with no traffic represents the least challenging environment that we have measured; it has the lowest observed median PLE $(\beta=3.10)$ and the lowest shadowing parameter $\left(\sigma_{d B}=3.23 \mathrm{~dB}\right)$. With our radio configuration, nodes were able to communicate when they were up to 750 meters apart.
Comparison with the heavy traffic scenario highlights the impact of environmental obstacles on signal attenuationin this case, vehicles that transiently impede LOS communication. Traffic causes an increase in median PLE (to $\beta=3.31)$ and significantly larger variability in signal strength $\left(\sigma_{d B}=4.84 \mathrm{~dB}\right.$ versus $\sigma_{d B}=3.23 \mathrm{~dB}$ with no traffic). The variability is caused by the presence/absence of vehicles blocking LOS communication at different distances and times.

At distances greater than 400 meters, the impact of traffic is lower because the vehicles were clustered around an intersection far from either of the nodes, and their apparent size (and the probability of blocking LOS communication) is much smaller when they are far away. At these large distances, the median PLE is approximately $\beta=3.1$, which is close to the value observed without traffic $(\beta=3.10)$. Also, the standard deviation in signal strength for packets at distances greater than 400 meters $\left(\sigma_{d B}=2.74\right)$ is similar to the shadowing parameter with no traffic $\left(\sigma_{d B}=3.23\right)$ and is much smaller than the standard deviation observed at distances less than 400 meters $\left(\sigma_{d B}=4.75\right)$.

Suburban. Figure 3(b) shows the distribution of LOS communication in our suburban environment. The median PLE, $\beta=3.14$, is similar to that of the open field environment with no traffic $(\beta=3.10$, Fig. 3(a)). There was little traffic when the measurements were taken, but the suburban environment's obstacles (e.g. buildings, parked vehicles, trees) result in significantly greater variation in signal propagation $\left(\sigma_{d B}=7.28\right)$ compared to the open field environment with no traffic $\left(\sigma_{d B}=3.23\right)$.

Urban. Figure 3(c) shows the LOS measurements from the most challenging of our three settings- the urban environment. The median PLE, $\beta=3.17$, is similar to that of the suburban environment $(\beta=3.14)$ and the open field without traffic $(\beta=3.10)$. However, numerous opportunities for reflection as well as a high incidence of other vehicles obstructing the LOS path give this environment the highest shadowing parameter $\left(\sigma_{d B}=9.15\right)$ of the LOS measurements. This implies that even under LOS conditions, communication in this environment will be comparatively less reliable because of the higher variability due to obstacles and intervening vehicles relative to the lower shadowing parameters in the open field (with traffic, $\sigma_{d B}=4.84$; without traffic, $\left.\sigma_{d B}=3.23\right)$ and suburban $\left(\sigma_{d B}=7.28\right)$ environments.

4.3.2. Around-the-Corner. In the following paragraphs we discuss the impact of the transition from LOS to non-LOS communication in each setting. In the suburban and urban environments, there are significant obstacles on the blocks between the nodes (e.g. buildings, trees). When the moving node is at the intersection, there is LOS communication between the nodes. However, as it travels away from the intersection and intervening obstacles block LOS communi- 


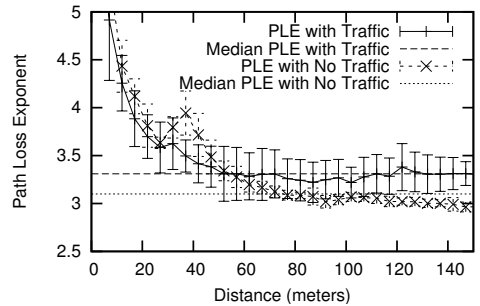

(a) Open Field

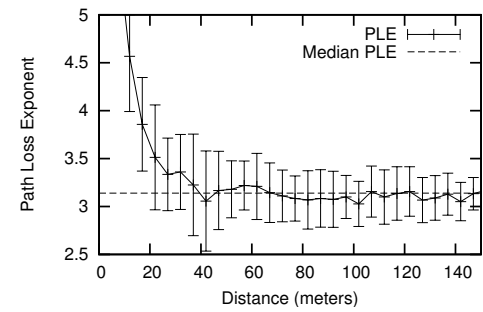

(b) Suburban

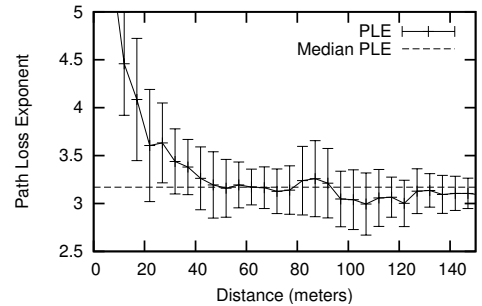

(c) Urban

Figure 3. LOS measurements with median path loss exponents. Error bars show standard deviation of PLE. For the open field case, we show measurements with and without traffic.

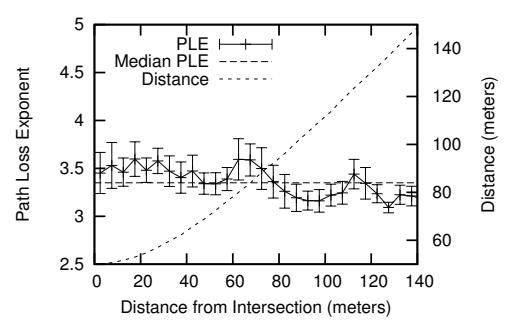

(a) Open Field

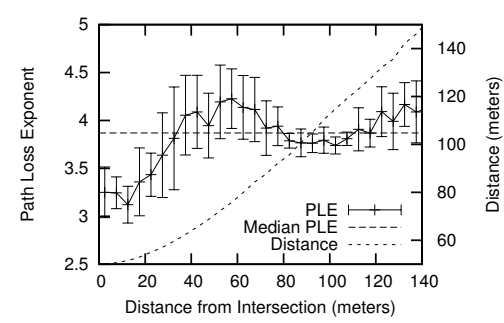

(b) Suburban

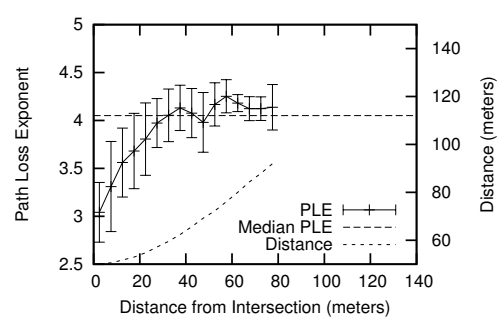

(c) Urban

Figure 4. ATC measurements with median path loss exponents. Error bars show standard deviation of PLE.

cation, we expect that the observed PLE will rise because obstacles will play an increasing role in attenuating the signal until that communication is only possible via a nonLOS (e.g. reflected) path. In all cases, the parked node was approximately 50 meters away from the intersection.

Open Field. Figure 4(a) shows the distribution of effective PLE at the given distance from the intersection under the open field with traffic scenario. The secondary curve (on the right axis) plots the absolute distance between the nodes as the distance of the moving node from the intersection increases. For example, when $x=40$ (the node is 40 meters from the intersection), the absolute distance between the nodes is just over 60 meters (the dashed curve is plotted on the right $y$-axis). At this distance the mean PLE (plotted on the left $y$-axis) is about 3.5, which is above the median PLE for the environment of 3.35 (drawn as a horizontal line).

As the node moves away from the intersection, the mean PLE does not deviate by more than 0.2 from the median value; this curve has the same shape as the open field LOS curves because there are no obstacles on the block of land that separates the vehicles, and therefore no significant signal attenuation.

Suburban. In our suburban setting, the change from LOS to non-LOS communication has a clear impact on the median PLE and shadowing parameter. Figure 4(b) shows that, unlike the open field environment, when the nodes are both 50 meters from the intersection, the mean PLE for that distance was $\beta=4.2$, which is significantly larger than the LOS PLE of 3.14. Greater variability due to changing ob- stacles results in larger standard deviations in RSSI (as great as $8.3 \mathrm{~dB}$ ) when the node is between 30 and 70 meters from the intersection; when the node is less than 30 meters from the intersection, the average standard deviation observed was much smaller, about $3.55 \mathrm{~dB}$. Changes in PLE, aside from the increase at low distances from the intersection, may be due to the signal having a more or less obstructed path; for example, the region of low PLE $(\beta=3.75)$ from 80 to 100 meters from the intersection could be a brief window of opportunity for the signal to pass behind the house that had initially blocked the LOS path (from 30 to 80 meters from the intersection).

Urban. Finally, we study the impact of around-the-corner communication in our example urban environment. The trend in Figure 4(c) is similar to that of the suburban aroundthe-corner experiment, but this scenario is even more challenging for communication. The urban around-the-corner measurements have the highest median PLE $(\beta=4.05)$ and shadowing $\left(\sigma_{d B}=10.74\right)$ parameters of all our experiments. In the suburban ATC experiment, nodes were able to communicate from as far as 150 meters away; however, the urban environment restricted ATC communication to only 90 meters, a $40 \%$ reduction in non-LOS transmission range.

Since the urban environment's buildings are closer to the street than in the suburban setting, the rise in PLE due to the obstructing building is more abrupt. PLE increases as soon as the node moves away from the intersection, and peaks $(\beta=4.25)$ at about 80 meters from the intersection; in comparison, the suburban ATC experiment showed that a 


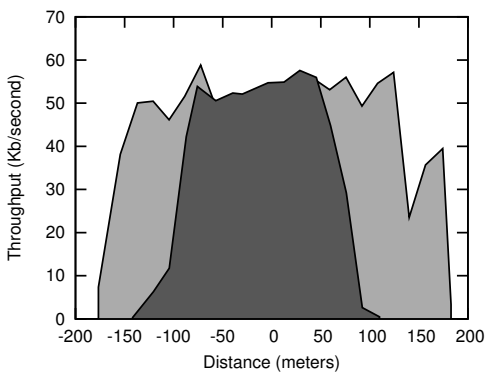

Figure 5. Throughput as a function of distance between two nodes in an urban environment. The light gray area shows LOS throughput, and the dark gray area shows ATC throughput. LOS and ATC conditions are described by Figure 6 .

node was able to travel about 20 meters from the intersection before seeing a significant rise in PLE (which then peaked at $\beta=4.2,60$ meters from the intersection).

A non-LOS path has lower signal strength and higher PLE than a LOS path because the signal travels a longer distance than the direct path and is attenuated (e.g. due to reflection, diffraction). This explains the increase in median PLE in our ATC suburban and urban experiments relative to LOS communication.

\section{Impact of More Realistic Models}

The variations in PLE and shadowing parameters across different environments and node placements should have a significant impact on the performance of an application. For example, Figure 5 shows the measured throughput for a single experiment run as a function of distance between two vehicles under the LOS and ATC cases. For this experiment, we adopted the same experimental configuration as in Section 4. As shown in Figure 6, we parked one node $(A)$ on the side of the road in the middle of a block. In the LOS experiment, $B$ drives on the same street to maintain LOS for the duration of connectivity. In the ATC experiment, $C$ drives around the block so that the effect of obstacles blocking LOS can be measured. The ATC trace shows lower throughput relative to the LOS trace at distances greater than $\sim 60$ meters, as $C$ turns at each of the corners.

To better understand the impact of more realistic signal propagation models, we evaluate a simple epidemic protocol under a variety of environments using the parameter settings derived from our measurements. The following paragraphs describe our evaluation and present an analysis of our key findings.

\subsection{Evaluation Setup}

We use the JiST/SWANS simulator [16], which provides an integrated, configurable, and flexible environment for

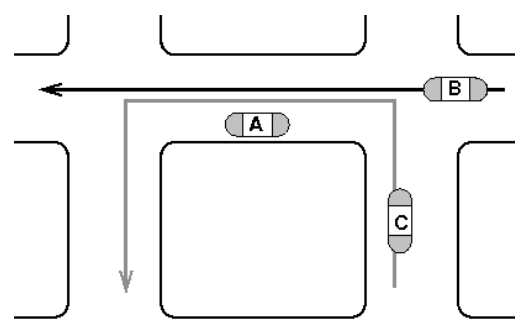

Figure 6. Experiment configuration for the throughput analysis. For LOS, $B$ drives past the parked node $(A)$, staying on the same street. For ATC, $C$ drives around the block of $A$.

evaluating ad hoc routing protocols, especially for largescale network scenarios. It contains a detailed model of the IEEE 802.11 wireless LAN protocol and a stochastic radio channel model, both of which we used in this work. The STreet RAndom Waypoint (STRAW) [13] mobility model captures realistic vehicular mobility, incorporating well-understood car-following and lane-changing models and traffic control, over actual city road maps imported from the TIGER/LINE database [22]. We evaluate the impact of environment using empirically determined path loss exponent and shadowing parameters.

\subsection{Epidemic Protocol}

For this analysis, we implemented a simple epidemic protocol following the model proposed by Vahdat and Becker in [23] and similar to Burgess et al. [24]. Such datadissemination protocols are fairly well-understood and are commonly used in this domain because they accommodate the frequent network disconnections that are observed in many vehicular networks.

Our protocol implementation has two phases of interaction: nodes send periodic beacon digests with a summary of messages in their buffer, and other nodes respond over unicast with data packets that the beaconing node has not yet received. A subset of nodes are transmitters, which periodically inject messages of a given size with a random destination.

To improve scalability to number of nodes and limit packet drops due to transmission synchronization we probabilistically control the number of nodes responding to beacons. Nodes respond to beacons with a probability inversely proportional to the number of beacons that were received in the last interval.

\subsection{Simulation Parameters}

All simulations are conducted on a map of the same region of Chicago, bounded on the east side by Lake Michigan and on the south by the Chicago River. The field extends about 
$1.6 \mathrm{Km}$ to the west and $1.1 \mathrm{Km}$ to the north, giving an area of about $1.76 \mathrm{Km}^{2}$. The area contains 331 road segments with a total length of $60 \mathrm{Km}$.

We place 150 nodes on the field at random intersections, and select 25 of these nodes to be transmitters. Each node sends a beacon on average every 5 seconds, and the transmitting nodes inject a message with average size of $10 \mathrm{~KB}$ approximately every 60 seconds. Total simulation time is 2 hours.

The parameters of the radios in simulation are configured to match the capabilities of the hardware used in our experiments. We specify the transmission power to be $26 \mathrm{dBm}$, give each node an antenna gain of $7 \mathrm{dBi}$, and fix the data rate at $2 \mathrm{Mbps}$.

\section{Analysis of the Impact of More Realistic Models}

Here, we discuss the impact of varying signal propagation model configuration with respect to application performance. The data shown represents the system's behavior after 2500 seconds have elapsed. This warm-up period was empirically determined to be the point at which the protocol reached a steady state, characterized by nodes' buffers reaching capacity and the packet drop rate stabilizing.

\subsection{Metrics}

Message delivery ratio and latency are the most straightforward methods of evaluating the application's performance. Figure 7 shows the CDF of latency from the time a message was posted until the time it was received at the destination node. The CDF curves do not reach 1 because not all messages were received at their destination.

We use the packet drop rate due to concurrent transmissions measured by the simulator as a metric to support our interpretation of the performance results. In Figure 8 we show the median and 3rd quartile of packet drop rate calculated from the distribution of the simulations' drop rate after the 2500 second warm-up period.

\subsection{Discussion}

Many studies assume an idealized open field environment, which can be sufficiently modeled by a single set of LOS signal propagation model parameters. The LOS and ATC parameters from our open field measurements were nearly identical because there were no obstacles to block LOS, so the median delivery latency (50.4 seconds), delivery ratio (98.8\%), and median packet drop rate (LOS: 42.8, ATC: 45 seconds) were approximately the same under these two conditions (Figure 7(a)).

In more complex environments, however, a simple set of parameters does not capture the effects of obstacles on signal propagation. Our measurements show that LOS was a significant factor in determining signal attenuation in urban and suburban settings. For example, in the urban environment, the difference in median PLE between LOS and ATC is 0.88, and the LOS and ATC shadowing parameters differ by 1.59 .

More complex approaches, such as ray-tracing by McKown and Hamilton [14], rely on a database containing the exact locations of all obstructions and terrain features to accurately model the signal propagation in the environment The received signal strength is determined through computation that accounts for each individual obstacle. While more realistic, such approaches are prohibitively expensive in terms of computation and clearly infeasible for experimentation with large scale VANETs.

Our studies suggest that there is a possible compromise solution that takes advantage of the two dominant classes of vehicular position configurations - in line-of-sight or around-the-corner. Using a basic shadowing model with logdistance path loss, this approach selects parameter settings according to the relative positions of the vehicles. We refer to this variation of the Uniform model as $L O S \& A T C$. This solution achieves a higher level of realism than the Uniform model without sacrificing scalability by taking advantage of environmental information, including road network layout and node position data.

It is clear that neither Uniform LOS nor Uniform ATC parameter settings will yield realistic results. Under the $L O S \& A T C$ configuration in the urban environment (Figure 7(c)), the median delivery latency is 65.8 seconds. In comparison, the median delivery latency under Uniform LOS is $77 \%$ shorter (15 seconds) than the LOS\&ATC latency, while the median delivery latency for Uniform ATC is $102 \%$ longer (133 seconds). The urban Uniform LOS and ATC configurations have at least a two-fold difference in median delivery latency from the $L O S \& A T C$ configuration.

A simple alternative would be to combine the measurements into a single set of parameters, which we label the Uniform Combined configuration. The shadowing parameter in this configuration is larger than the values for either Uniform LOS or ATC. This is because combining the different signal propagation effects of LOS and ATC communication in complex (e.g. suburban, urban) environments results in greater standard deviation to accommodate the variation than would be necessary to model one of the distributions separately. For example, in the suburban environment, the shadowing parameter is $\sigma_{d B}=7.28$ for LOS and 8.44 for ATC, but 9.40 for Combined. The median PLE, on the other hand, is an average of the LOS and ATC values; in the suburban environment, the Combined PLE is $\beta=3.62$, between the LOS (3.14) and ATC (3.87) values. This results in comparatively lower communication performance (relative to the real world) when vehicles are in LOS because the Combined PLE is more restrictive (larger) than the LOS PLE and reduces the transmission radius. On the other hand, this 


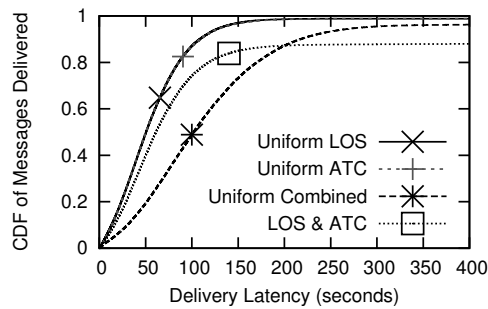

(a) Open Field

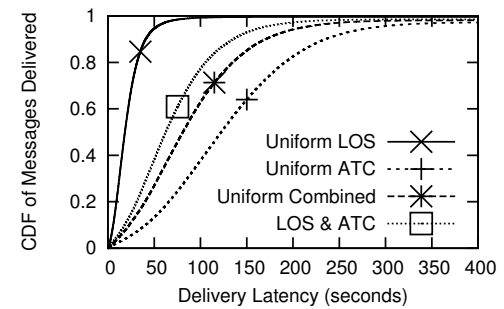

(b) Suburban

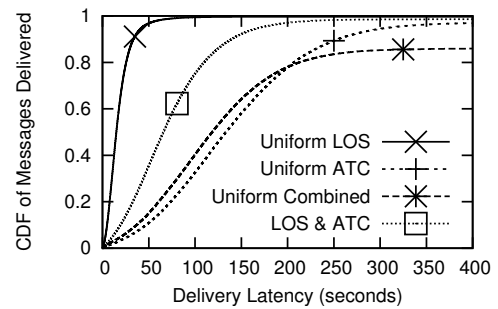

(c) Urban

Figure 7. CDF of delivery latency for open-field, suburban and urban settings.

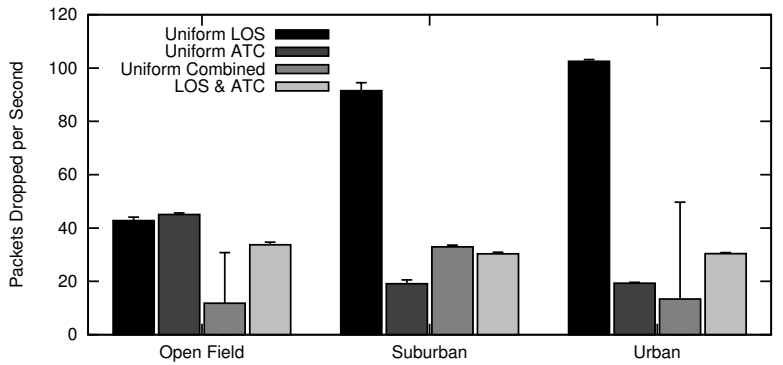

Figure 8. Median and 3rd quartile of packet drop rate.

results in higher communication performance than would be observed in the real world when vehicles are in ATC because the Combined PLE is smaller and less restrictive than ATC PLE.

Overall, the Uniform Combined configuration results in worse performance than $L O S \& A T C$ in the suburban and urban environments. In the urban setting, the median delivery latency under the Uniform Combined configuration is $80 \%$ greater than that of the LOS\&ATC configuration, and the Combined delivery ratio is $11 \%$ lower than under $L O S \& A T C$. The higher shadowing parameter makes communication between two nodes at any distance less reliable. Also, the averaged PLE penalizes LOS communication while permitting too much communication between nodes on different streets, leading to higher noise levels and increased packet drops (especially in the urban environment, where the third quartile of Combined drop rate is $61 \%$ greater than the third-quartile $L O S \& A T C$ drop rate).

\section{Related Work}

Opportunistic vehicle-to-infrastructure communication (e.g. between IEEE 802.11 mobile nodes and access points) is a viable model, given that the system can tolerate periods of network disconnection due to the limited distribution of available access points. Experiments in [25] showed that a vehicle can opportunistically connect to a wireless network and exchange data even when traveling at high speed. Measurement studies of vehicular opportunistic con- nectivity have analyzed the impact of IEEE 802.11 MAC bitrate selection algorithms on TCP goodput [26] and used the CarTel deployment to survey available access points, connection latency, throughput and drop rate [27].

Vehicle-to-vehicle VANET communication has limitations similar to those of the opportunistic model, including periods of disconnection due to the distribution of instrumented vehicles. Measurement studies in this context have analyzed the impact of LOS communication in varied environments [28], and noted that maintaining LOS is a significant factor in communication [29]. Another study of single- (I2V) and multi-hop (I2V/V2V hybrid) communications conducted on a highway specifically calls for analysis of factors such as environment, weather, and other vehicles in signal propagation [30]. Other work characterized propagation in open field and highway environments for the DSRC band using the Nakagami model [31].

\section{Conclusion}

This paper presented a study of the impact of realistic radio propagation settings on the evaluation of VANETbased systems. Through an experimental study with instrumented vehicles in a variety of urban and suburban environments we studied how signal propagation varies in different settings, especially between line-of-sight (LOS) and around-the-corner (non-LOS) communication. Based on this study, we evaluated the impact of different empirical parameter settings on the performance of a basic epidemic data dissemination protocol and discuss the implications of our findings. As part of this work, we suggested a possible approach that incorporates additional realism in signal propagation models for high-level simulators without sacrificing scalability by taking advantage of environmental information, including node locations and street information.

While our epidemic protocol implementation is simple, leaving room for optimization, our goal is to understand the impact of more realistic signal propagation models on application performance. These effects are independent of implementation and generalizable to other applications. The design of new data dissemination protocols based on these new insights is part of our future work. 


\section{References}

[1] B. Hoh, M. Gruteser, R. Herring, J. Ban, D. Work, J.C. Herrera, A. Bayen, M. Annavaram, and Q. Jacobson, "Virtual trip lines for distributed privacy-preserving traffic monitoring," in Proc. of ACM/USENIX MobiSys, 2008.

[2] B. Hull, V. Bychkovsky, Y. Zhang, K. Chen, M. Goraczko, A. Miu, E. Shih, H. Balakrishnan, and S. Madden, "CarTel: a distributed mobile sensor computing system," in Proc. of ACM SenSys, 2006.

[3] H. Zhu, Y. Zhu, M. Li, and L. M. Ni, "HERO: online real-time vehicle tracking in Shanghai," in Proc. of IEEE INFOCOM, 2008 .

[4] M. D. Dikaiakos, S. Iqbal, T. Nadeem, and L. Iftode, "VITP: an information transfer protocol for vehicular computing," in Proc. of ACM VANET, 2005.

[5] J. Yoon, B. Noble, and M. Liu, "Surface street traffic estimation," in Proc. of ACM/USENIX MobiSys, 2007.

[6] U. Lee, E. Magistretti, B. Zhou, M. Gerla, P. Bellavist, and A. Corradi, "MobEyes: smart mobs for urban monitoring with vehicular sensor networks," IEEE Wireless Communications, October 2006

[7] K. Pawlikowski, H.-D. J. Jeong, and J.-S. R. Lee, "On credibility of simulation studies of telecommunication networks," IEEE Communications, January 2002.

[8] T. R. Andel and A. Yasinac, "On the credibility of Manet simulations," IEEE Computer, July 2006.

[9] D. Kotz, C. Newport, R. S. Gray, J. Liu, Y. Yuan, and C. Elliott, "Experimental evaluation of wireless simulation assumptions," in Proc. of ACM MSWIM, 2004.

[10] M. Takai, J. Martin, and R. Bagrodia, "Effects of wireless physical layer modeling in mobile ad hoc networks," in Proc. of ACM MobiHoc, 2001.

[11] G. Zhou, T. He, S. Krishnamurthy, and J. A. Stankovic, "Impact of radio irregularity on wireless sensor networks," in Proc. of ACM/USENIX MobiSys, 2004.

[12] V. Naumov, R. Baumann, and T. Gross, "An evaluation of inter-vehicle ad hoc networks based on realistic vehicular traces," in Proc. of ACM MobiHoc, 2006.

[13] D. R. Choffnes and F. E. Bustamante, "An integrated mobility and traffic model for vehicular wireless networks," in Proc. of ACM VANET, 2005.

[14] J. W. McKown and R. L. Hamilton, "Ray tracing as a design tool for radio networks," IEEE Network Magazine, November 1991.

[15] J. Rybicki, B. Scheuermann, W. Kiess, C. Lochert, P. Fallahi, and M. Mauve, "Challenge: peers on wheels - a road to new traffic information systems," in Proc. of ACM/IEEE MobiCom, 2007.
[16] R. Barr, "An efficient, unifying approach to simulation using virtual machines," Ph.D. dissertation, Cornell University, 2004.

[17] T. Rappaport, Wireless communications: Principles and Practice, 2nd ed. Prentice Hall, 2002.

[18] S. McCanne and S. Floyd, "ns Network Simulator," http:// www.isi.edu/nsnam/ns/.

[19] L. Bajaj, M. Takai, R. Ahuja, and R. Bagrodia, "Simulation of large-scale heterogeneous communication systems," in Proc. of MILCOM, October 1999.

[20] W. C. Y. Lee, Mobile Communicaitons Engineering. McGraw-Hill, 1982.

[21] L. Cheng, B. Henty, D. D. Stancil, F. Bai, and P. Mudalige, "A Fully Mobile, GPS Enabled, Vehicle-to-Vehicle Measurement Platform for Characterization of the $5.9 \mathrm{GHz}$ DSRC Channel," in IEEE AP-S, 2007.

[22] "U.S. Census Bureau - TIGER/LINE," http://www.census. gov/geo/www/tiger/.

[23] A. Vahdat and D. Becker, "Epidemic routing for partially connected ad-hoc networks," Duke University, Tech. Rep., 2000 .

[24] J. Burgess, B. Gallagher, D. Jensen, and B. N. Levine, "MaxProp: Routing for Vehicle-Based Disruption-Tolerant Networks," in Proc. of IEEE INFOCOM, 2006.

[25] J. Ott and D. Kutscher, "Drive-thru Internet: IEEE $802.11 \mathrm{~b}$ for "Automobile" Users"," in Proc. of IEEE INFOCOM, 2004.

[26] D. Hadaller, S. Keshav, T. Brecht, and S. Agarwal, "Vehicular opportunistic communication under the microscope," in Proc. of ACM/USENIX MobiSys, 2007.

[27] V. Bychkovsky, B. Hull, A. Miu, H. Balakrishnan, and S. Madden, "A measurement study of vehicular Internet access using in situ Wi-Fi networks," in Proc. of ACM/IEEE MobiCom, 2006.

[28] J. Singh, N. Bambos, B. Srinivasan, and D. Clawin, "Wireless LAN Performance Under Varied Stress Conditions in Vehicular Traffic Scenarios," in Proc. of IEEE VTC, 2002.

[29] M. Jerbi, P. Marlier, and S. Senouci, "Experimental Assessment of V2V and I2V Communications," IEEE MASS, 2007.

[30] H. Wu, M. Palekar, R. Fujimoto, R. Guensler, M. Hunter, J. Lee, and J. Ko, "An empirical study of short range communications for vehicles," in Proc. of ACM VANET, 2005.

[31] V. Taliwal, D. Jiang, H. Mangold, C. Chen, and R. Sengupta, "Empirical determination of channel characteristics for DSRC vehicle-to-vehicle communication," in Proc. of ACM VANET, 2004. 